\title{
PREMEDICATION PROTOCOLS IN DENTAL PRACTICE IN ALLERGIC PATIENTS
}

\author{
Angelina Kisselova ${ }^{1}$, Adriana Krasteva ${ }^{2}$, Assya Krasteva ${ }^{1}$ \\ 1) Department of Imaging and Oral Diagnostic, Faculty of Dental Medicine, \\ Medical University, 1 G. Sofiyski Blvd., Sofia 1000, Bulgaria \\ Medical Center "Detsko zdrave”, 27-29 Ami Bue Str., Sofia 1000, Bulgaria \\ 2)Department of Pharmacology and Toxicology, Faculty of Pharmacy, Medical \\ University, 2, Dunav str. Sofia 1000, Bulgaria
}

\section{ABSTRACT}

The problem with choosing a suitable pre-medication protocols before local anesthesia in dentistry in allergic patients is always discussed, as in the dental practice different schemes are already proven $(3,5)$. The propose of this communication is to share the experience on those pre-medication schemes in allergic patients during and outside pollen season.

Key words: Pre-medication protocols, allergic patients, dental anesthesia

\section{INTRODUCTION}

To be considered safe, dental treatment (including usually local anaesthesia) needs some precautions which sometimes are neglected by dental practitioners. Obtaining a credible medical history is the first step, especially in allergic patients. An examination by an allergist and skin testing is needed. Based on this information a premedication
\end{abstract}

protocol will be administrated and this decision is repeatedly discussed by a number of bulgarian doctors ( 1 , $2,3,4,5,6)$.

\section{THE AIM:}

Our aim is to share the experience on those premedication schemes in allergic patients during and outside pollen season.

As in non-allergic patients, the premedication regimens in allergic people include the same medications however the difference appears in posology and treatment duration. It should be noted that in allergic patients anaesthetic dental procedures should be avoided during the pollen season, especially if they have had a former serious allergic reaction (4).

Two pre-medication schemes are proposed to reduce the risk of severe allergic reaction during a dental procedure.

Table 1. Premedication protocols in allergic patients with negative skin testing for hypersensitivity to local anaesthetics

\begin{tabular}{|l|l|l|}
\hline Drugs & & Premedication scheme \\
\hline H1 blockers: & & \\
Cetirizine hydrochloride (Zyrtec) & $10 \mathrm{mg} /$ daily & 5 days prior and 5 days after dental procedure \\
Levocetirizine hydrochloride (Xyzal) & $5 \mathrm{mg}$ daily & \\
Loratadine (Claritine, Roletra) & $10 \mathrm{mg}$ daily & \\
Desloratadine (Aerius) & $5 \mathrm{mg}$ daily & \\
Fexofenadine (Telfast, Ewofex) & $120 \mathrm{mg} / 180 \mathrm{mg}$ daily & \\
\hline H2 blockers: & & \\
Ranitidine hydrochloride (Ranitidine, Zantac) & $300 \mathrm{mg}$ daily & 5 days prior and 5 days after dental procedure \\
Famotidine (Quamatel, Famotidine) & $40 \mathrm{mg}$ daily & \\
\hline Vitamins: & $1000 \mathrm{mg}$ daily & 5 days prior and 5 days after dental procedure \\
Ascorbic acid (Vitamin C) & & \\
\hline Corticosteroids: & $4 \times 5 \mathrm{mg}$ daily & 5 days prior and 5 days after dental procedure \\
Prednisolone tabl.
\end{tabular}

*The suggested protocols are adapted for children older than 6 years. 
Table 2. Premedication protocols in allergic patients with positive skin testing for hypersensitivity to local anaesthetics

\begin{tabular}{|c|c|c|}
\hline Drugs & & Premedication scheme \\
\hline \multicolumn{3}{|l|}{ H1 blockers: } \\
\hline Cetirizine hydrochloride (Zyrtec) & $10 \mathrm{mg} /$ daily & 7 days prior and 7 days after dental procedure \\
\hline Levocetirizine hydrochloride (Xyzal) & $5 \mathrm{mg}$ daily & \\
\hline Loratadine (Claritine, Roletra) & 10 mg daily & \\
\hline Desloratadine (Aerius ) & 5 mg daily & \\
\hline Fexofenadine (Telfast, Ewofex) & $120 \mathrm{mg} / 180 \mathrm{mg}$ daily & \\
\hline \multicolumn{3}{|l|}{ H2 blockers: } \\
\hline Ranitidine hydrochloride (Ranitidine, Zantac) & $300 \mathrm{mg}$ daily & 7 days prior and 7 days after dental procedure \\
\hline Famotidine (Quamatel, Famotidine) & $40 \mathrm{mg}$ daily & \\
\hline \multicolumn{3}{|l|}{ Vitamins: } \\
\hline Ascorbic acid (Vitamin C) & $1000 \mathrm{mg}$ daily & 7 days prior and 7 days after dental procedure \\
\hline \multicolumn{3}{|l|}{ Corticosteroids: } \\
\hline Prednisolone tabl. & 4 x 5 mg daily & 5 days prior and 5 days after dental procedure \\
\hline $\begin{array}{l}\text { Methylprednisolone sodium succinate } \\
\text { (Solu-Medrol) }\end{array}$ & $\begin{array}{l}40-60 \mathrm{mg} \text { IM } \\
\text { (according to patients } \\
\text { body weight) }\end{array}$ & 1 hour prior the dental procedure \\
\hline
\end{tabular}

*The suggested protocols are adopted for children older than 6 years.

\title{
CONCLUSION
}

A precaution must always be taken during the dental treatment of allergic patients in order to achieve satisfactory results by avoiding serious allergic reaction or even anaphylactic shock.

\section{REFERENCES:}

1. Djerasi, E., B. Petrunov. Alergologichni problem v stomatologijata. publisher medizina i fiskultura, Sofia, 1990, 245. (in Bulgarian)

2. Kisselova-Yaneva, A. Lokalni uslojnenija i inzidenti pri prilagane na lokalni anestetizi. Godishen sbornik na IMAB, 2002, 8(1):76-77. (in Bulgarian)
3. Kisselova-Yaneva, A. Stomatologichna alergologia i ognistna diagnostic. Monograph, publisher "Gutenberg", Sofia, 2001, 327. (in Bulgarian)

4. Mileva, J. Klinichna alergologia ( in bulgarien). Book, publisher "Znanie", Sofia, 2001. (in Bulgarian)

5. Petrunov B., A. Kisselova-Yaneva,
V. Dimitrov et al. Klinichna imunologija, klinichna alergologija, dentalna klinichna alergologija. Book, publisher "Arso", Sofia,., 2009. (in Bulgarian)

6. Mileva, J. Savremeno lechenie na alergichnite bolesti. Book, publisher "Znanie", Sofia, 1999. (in Bulgarian)

\author{
Adrress for correspondence: \\ Angelina Kisselova \\ Department of Oral imaging and oral diagnostic, Faculty of Dental Medicine, \\ Medical University - Sofia \\ 1, Sveti Georgi Sofiiski Blvd., 1000 Sofia, Bulgaria \\ E-mail: prof_kisselova@yahoo.com
}

